\title{
Micro-moulded substrates for the analysis of structure-dependent behaviour of nematodes
}

\author{
Kazunori Oтове *, Kenji Itou and Takayuki Mizukuвo \\ National Agricultural Research Center, National Agricultural Research Organization, 3-1-1 Kannondai, Tsukuba, \\ Ibaraki 305-8666, Japan
}

Received: 2 October 2003

Accepted for publication: 7 November 2003

\begin{abstract}
Summary - Microstructures, consisting of networks of channels of rectangular cross sections (50 $\mu \mathrm{m}$ high, 40-200 $\mu \mathrm{m}$ wide), were formed in $5 \mathrm{~mm}$ square areas on transparent substrates made of silicon rubber. An experimental set-up using the network sealed with a flat glass plate has the potential to function in a way similar to the pore space in soil, and is therefore useful for studies of nematode migration. The set-up allowed the migratory activity of nematodes in water-filled, porous and transparent microstructuresto be observed with a microscope. By means of substrates with two different channel dimensions, the structure-dependent behaviour of second-stage juveniles of Meloidogyne incognita was visually demonstrated. Their behaviour was examined on the basis of the migration patterns obtained by superimposing recorded serial images of individual juveniles. In a micro-channel network with $40 \mu \mathrm{m}$ high channels of $200 \mu \mathrm{m}$ wide elements, juveniles showed marked activity in migration, forming consistent zigzag patterns spread over the network area. In contrast, in a micro-channel network with $80 \mu \mathrm{m}$ high channels of $400 \mu \mathrm{m}$ wide elements, migration showed thick, sparse patterns, restricted around the area where the juveniles were initially deposited. This comparison showed that $M$. incognita juveniles in a narrow, fine network tended to migrate actively and, in contrast, those in a wide, coarse network were prevented from migrating by the network configuration.
\end{abstract}

Keywords - Meloidogyne incognita, microchannel network, migration, pore space.

The behaviour of plant-parasitic nematodes in soil is of considerable practical concern since these organisms affect the interactions between host plants and their environment. For the last decade, a habitable pore space hypothesis has been proposed, stating that soil animals (nematodes and protozoa) can only access water-filled pores with diameters greater than their body diameter (Hassink et al., 1993; Killham et al., 1993; Görres et al., 1999). From correlation analyses of pore size distributions of soils and the biomass of nematodes, the neck diam. of the habitable pore space were estimated as 30-90 $\mu \mathrm{m}$ (Hassink et al., 1993). Regarding plant-parasitic nematodes, Sano and Nakasono (1997) reported that Meloidogyne incognita second-stage juveniles tended to migrate gradually into soil aggregates during storage in the soil. As is suggested by the hypothesis and the reports above, the microstructures of soil aggregates where nematodes live may play an important role in their survival.

To validate the suggestion mentioned above, structural dependence and chemotaxis, involved in nematode be- haviour in pore space, have to be separately investigated. Conventionally, in vitro behaviour of nematodes has been studied using gel plates and sand columns; for example, reactions of nematodes to carbon dioxide (Pline \& Dusenbery, 1987; Robinson, 1995), temperature (Robinson, 1994) and antibodies (Sharon et al., 2002). Prior to these studies, knowledge of nematode behaviour was summarised by Croll (1970). There have been a few direct approaches to observe the relationship between nematode behaviour and soil structure using Petri dishes in which single layers of soil particles were formed (Wallace, 1958a, b). Indeed, using raw soils is practical to investigate the behaviour in pore space, but soil particles influence nematode behaviour because of their irregular shapes and, in addition, the irregularity prevents the soil structure from forming reproducibly. Thus, media consisting of adjustable transparent porous structures would be helpful for direct observations in experiments.

Recent progress in engineering has led to the development of $\mu \mathrm{m}$-sized machining technologies that have

*Corresponding author, e-mail: otobek@affrc.go.jp 
Table 1. Dimensions ( $\mu \mathrm{m}$ ) of the types of elements used in micromoulds to provide two substrates with different pore structures.

\begin{tabular}{lccc}
\hline & \multicolumn{3}{c}{ Dimension } \\
\cline { 2 - 4 } Element & $\mathrm{a}$ & $\mathrm{b}$ & $\mathrm{c}$ \\
\hline Type A & 100 & 40 & 50 \\
Type B & 200 & 80 & 100 \\
\hline
\end{tabular}

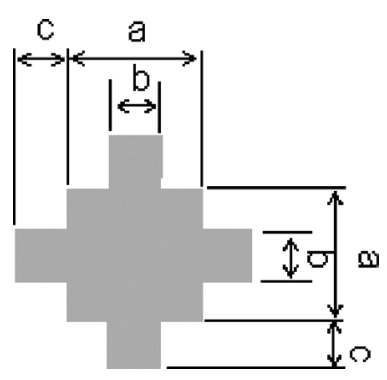

enabled us to fabricate microstructures with properties similar to soil-like media. Micro-moulding techniques (Brenner et al., 1993) (one of the micro-machining technologies) allow for the fabrication of $\mu \mathrm{m}$-sized channel networks on a small, transparent substrate. By means of artificial micro-channel networks with dimensional variation, we demonstrate in this report the structure-dependent behaviour of plant-parasitic nematodes without chemical influence. The difference in the behaviour recorded in video images is visually shown by image processing to extract the tracks of nematode migration in the networks.

\section{Materials and methods}

Meloidogyne incognita Omei strain was grown on tomato plants in a glasshouse. Several hundred newly hatched second-stage juveniles (J2) were obtained from egg masses collected from tomato roots. The $\mathrm{J} 2$ were placed in distilled water in a glass vessel, at ca $200 / \mathrm{ml}$, and stored at $10^{\circ} \mathrm{C}$ until use.

To obtain precisely formed micro-channel networks, two micro-moulds made of nickel alloy (50 $\mu \mathrm{m}$ thick and $10 \mathrm{~mm}$ square) were made using an electroforming technique, and then fixed on flat glass plates. An electroformed pattern of each micro-mould was made in an approximately $5 \mathrm{~mm}$ square at the centre, consisting of cross-shaped elements with the dimensions shown (Table 1). Elements were arranged by covering the square area but the element dimensions of the two micro-moulds were made differently. Polydimethylsiloxane (PDMS), a type of silicone used for moulding, was used to form substrates in the micro-moulds: 0.2 g PDMS was deposited and spread on a glass plate on which the micro-mould was fixed. The mould was left to cure overnight at room temperature and then peeled from the glass plate. The result was a chemically inactive, elastic, and transparent substrate, $26 \mathrm{~mm}$ diam. and $0.6 \mathrm{~mm}$ thick. A hydrophilic surface was formed on the substrate by sonicating it in a 2-methacryloyloxy ethyl phosphorylcholine aqueous solution $(5 \% \mathrm{v} / \mathrm{v})$ for $5 \mathrm{~min}$ and then rinsing with distilled water. The substrate could be used repetitively after cleaning by sonication in the solution.

This technique provided micro-moulded substrates with two different dimensions of micro-channel networks: one had micro-channels $40 \mu \mathrm{m}$ wide with $200 \mu \mathrm{m}$ elements (Type A) and the other had micro-channels $80 \mu \mathrm{m}$ wide with $400 \mu \mathrm{m}$ elements (Type B). In detail, the microchannel network of the type-A substrate consisted of channels with rectangular cross section $40 \mu \mathrm{m}$ wide, $50 \mu \mathrm{m}$ deep and $100 \mu \mathrm{m}$ long. At each channel intersection, a $100 \mu \mathrm{m}$ square with the same depth was made. The micro-channels of the type-B substrate were configured to be $80 \mu \mathrm{m}$ wide, $50 \mu \mathrm{m}$ deep and $200 \mu \mathrm{m}$ long, with a $200 \mu \mathrm{m}$ square at each intersection. The micro-moulded area that attached to the micro-mould surface was made on the substrates, set $50 \mu \mathrm{m}$ lower than the substrate surface that attached to the glass plate where the micromould was fixed. Other than in the micro-moulded area, the substrate surface closely contacted the glass plate, preventing nematodes escaping from the micro-moulded area when the micro-moulded side was placed on a flat substrate.

Two $\mathrm{ml}$ distilled water was poured into a well $(36 \mathrm{~mm}$ diam., $18 \mathrm{~mm}$ deep) of a cell-culture multi-dish, and a micro-moulded substrate was placed inside with the moulded side facing upward. A quartz cylindrical weight (20 mm diam., $5 \mathrm{~mm}$ thick) was placed on top of the substrate to cover the micro-channels. All bubbles on the substrate were removed to avoid obstructing nematode movement in the micro-channels. The dish was placed on the stage of an inverted microscope and $5 \mu 1$ nematode suspension were poured through an aperture $(2 \mathrm{~mm}$ diam.) in the centre of the cylindrical weight (Fig. 1A). The nematodes sank onto the micro-moulded side of the substrate and gradually migrated between the microchannels and the weight (Fig. 1B).

Migratory activity at $25^{\circ} \mathrm{C}$ was observed by transmitted light microscopy and by phase contrast microscopy, 

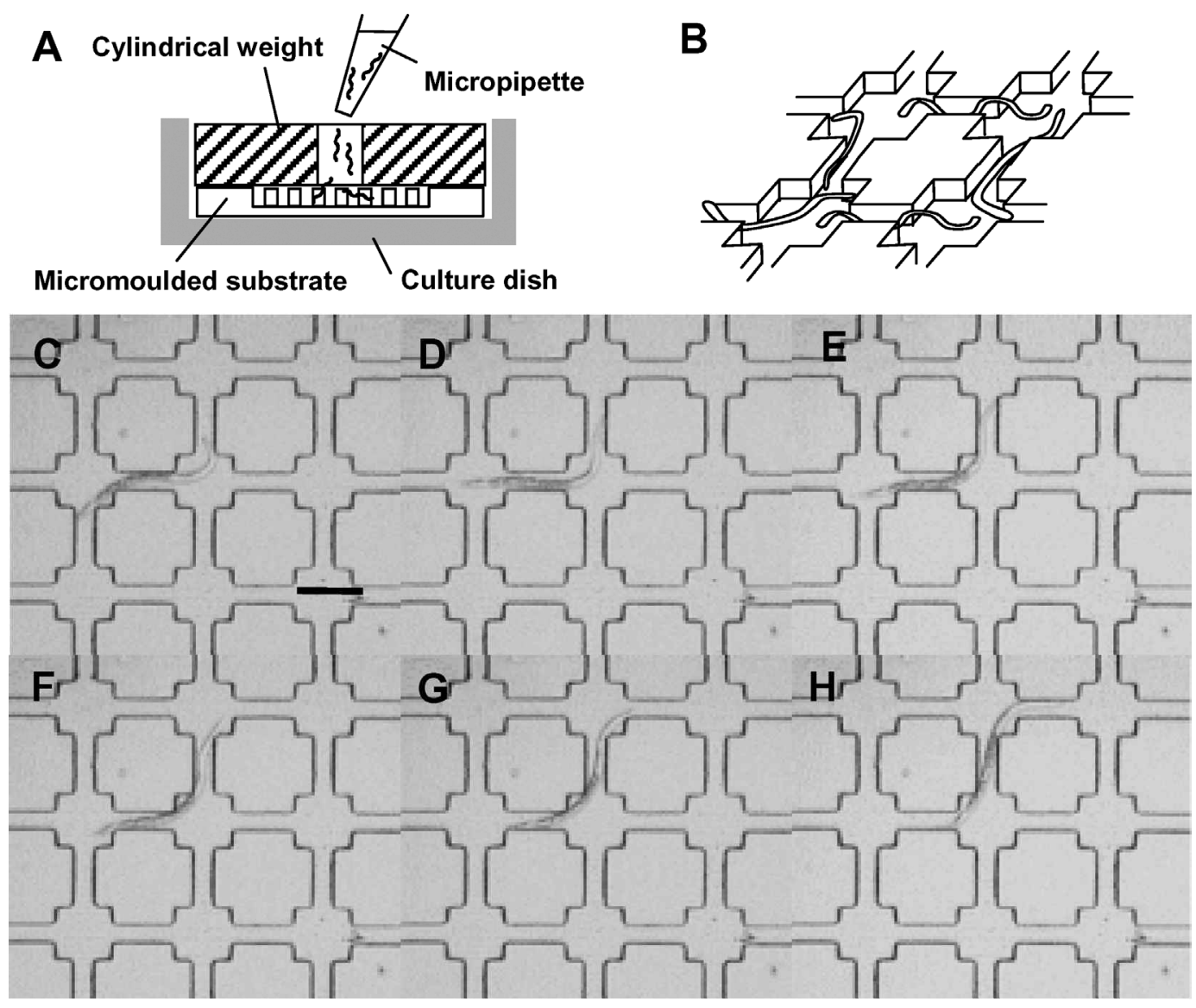

Fig. 1. Observation of migration characteristics of a Meloidogyne incognita second-stage juvenile (J2) in a micro-channel network produced using the micro-moulding technique. A: Schematic diagram of the experimental set-up; B: Illustration of J2 behaviour within the network (50 $\mu \mathrm{m}$ deep) covered with a cylindrical weight; $C$-H: Serial images of J2 migration upwards from left to right of each image using the sidewalls of the channels and exhibiting body-wiggling (scale bar $=100 \mu \mathrm{m}$ ).

recorded via a high-sensitivity CCD camera as microscope video images. The light source was set at faint intensity since the camera required only a little light and this helped to avoid irradiation to the nematodes observed. In addition, phase contrast microscopy provided highlighted, bright images of nematodes on a dark background of the micro-moulded substrates and this facilitated image processing. The video images observed by phase contrast microscopy were processed by differential image extraction and this processing provided still images where the tracks of the individual $\mathrm{J} 2$ in the micro-channel networks were superimposed. The duration of the video images processed was $10 \mathrm{~min}$. The video images were preliminarily converted to sequential still images with an interval of $1 \mathrm{~s}$ before the extraction process.

\section{Results}

After being deposited on the micro-moulded side of the type-A network, J2 exhibited free-waving activity since the micro-channels beneath the aperture were too shallow to hinder their motion. Although the waving itself hardly contributed to migration because of slippage in the water solution, occasional nematode displacement resulted from accidental contacts with the uncovered micro-channels during waving. The waving tended to be activated in response to intense light irradiation or vibration.

Some J2 used the micro-channels to thrust their anterior ends (heads) into the network sandwiched between the substrate and weight. By swinging the part of their body remaining in the aperture, they gradually advanced into 


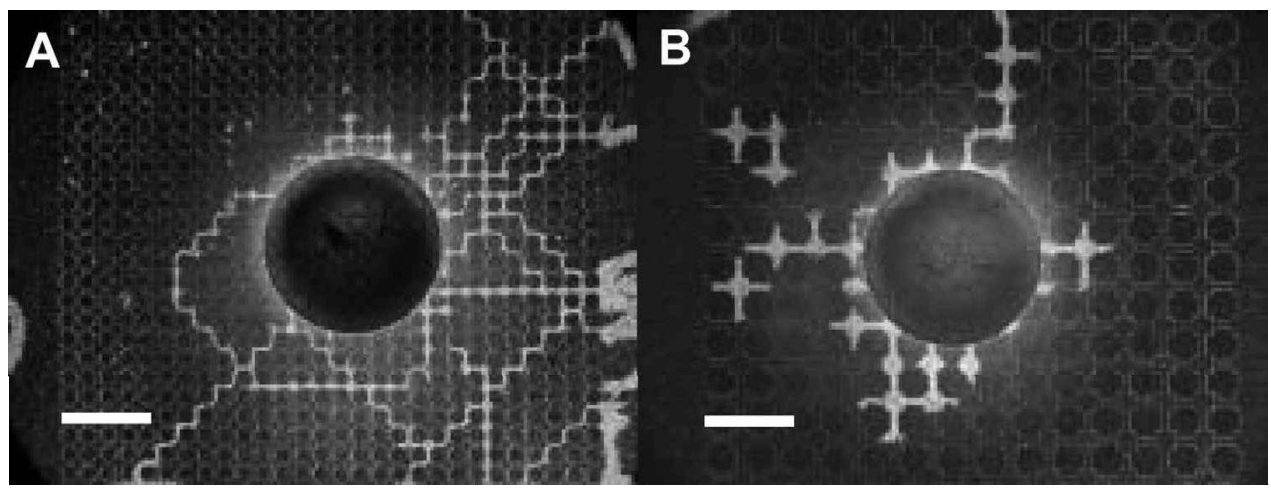

Fig. 2. Migratory activities of Meloidogyne incognita second-stage juveniles (J2) after deposition in micro-channel networks, represented by superimposed images of migration tracks for 10 min (bright lines). A: Activities of nine J2 in a type-A network; B: Activities of $14 \mathrm{~J} 2$ in a type-B network (scale bars $=1 \mathrm{~mm}$ ).

the sandwiched area that was significantly wider than the body diam. Such escape from the aperture area was assumed to be a random process since no attractant or repellent was introduced in the network.

Once J2 had entirely migrated throughout the sandwiched area, they moved their bodies by pushing against the sidewalls of the micro-channels, simultaneously wiggling their bodies (Fig. 1C-H). When J2 reached the intersection, their head swung from side to side, pressing onto sidewalls encountered, so that by wriggling their bodies slightly, they pushed against the other sidewalls their bodies were touching. This motion facilitated the advancement of their body toward the other intersection and repetition of this behaviour resulted in the migration of nematodes throughout the network.

The behaviour described above was commonly observed in both types A and B networks, while there was an obvious difference in the superimposed tracks which were represented as bright, thick lines in the superimposed photographs (Fig. 2A, B). In type-A networks, nine J2 formed consistent zigzag patterns spread over the network area, and showed marked activity in their migration. The superimposed video images clarified that $\mathrm{J} 2$ rhythmically and continuously migrated in the network. The continuous migration mostly resulted either in entrance to the aperture area or in escape from the network area and, consequently, few $\mathbf{J} 2$ remained in the network.

In contrast, $14 \mathrm{~J} 2$ migrating in the type-B network and showing thicker, sparser patterns than those in type A were restricted around the area where they were initially deposited. The thick lines showed that several wavings occurred at the same location. Furthermore, the sparse patterns showed that the length of individual migration tracks during 10 min was rather shorter than that in the type-A network. Although more J2 were introduced than in the type-A network, the spread of the resultant migration patterns in the network area was significantly smaller. This shows that the waving did not lead to migration in the type-B network and, as a result, $\mathrm{J} 2$ tended to remain in the network.

\section{Discussion}

The comparison of the migratory activities showed that $\mathrm{J} 2$ in a narrow, fine-pore space, filled with water tended to migrate actively but those in a wide, coarsepore space were prevented from migrating by the network configuration. This corresponds to the movement analyses conducted by Wallace (1958b), which revealed that there is a structural dependence in the migratory activity of nematodes. In his report, it was shown that both too large and too small particle sizes reduced the migration speeds of deposited Heterodera schachtii J2. In our experiment, the type-A network is considered to represent particles of $c a 230 \mu \mathrm{m}$ diam. and type-B particles of $450 \mu \mathrm{m}$ diam. Furthermore, the size of $M$. incognita J2 (15 $\mu \mathrm{m}$ body diam., $420 \mu \mathrm{m}$ long) is similar to that of $H$. schachtii $\mathrm{J} 2$ (18 and $480 \mu \mathrm{m}$, respectively). Thus, the same mechanism may be involved in the prevention of migratory activity. As Wallace (1958b) also mentioned, it is hypothesised that the wavelength of $\mathrm{J} 2$ waving was in harmony with the channel length of the type-A network and, consequently, efficient migration occurred. Artificial micro-channel networks can be used to accumulate consistent, explicit knowledge on this 
hypothesis by arbitrarily varying the arrangement and dimensions.

From the habitable pore space hypothesis, nematode preference for particular spaces can be assumed. The pore diameters employed in our experiments correspond to the pore-space sizes that are habitable for nematodes (Hassink et al., 1993). However, our result indicated that a water-filled pore space that allowed greater mobility of $M$. incognita $\mathrm{J} 2$ reduced the numbers remaining in the space. It is clear that $\mathrm{J} 2$ evasion did not cause this reduction since unforced entrance of $\mathrm{J} 2$ into the space, both from the outside and from the aperture area, was occasionally observed. The result suggests that reduced mobility of plant-parasitic nematodes in pore space promotes aggregation and that such pores seem to be habitable for the nematodes.

In our experimental set-up, the pore networks were simply spread in two-dimensional space while pore structure in soil is a three-dimensional network of pore chambers connected by constrictions (Glasbey et al., 1991). In addition, the arrangement and dimensions of pores are regular and thus different from raw soil structures. However, we consider that an entirely controllable physical environment for experiments is helpful since it can provide consistent, reproducible results. By means of artificial microchannel networks and introducing different nematodes, the mechanisms that form habitable pore spaces for nematodes may be revealed. This will contribute to reducing infection of roots by plant-parasitic nematodes. Our future research will furthermore focus on the influence of a range of physical, chemical, and electrochemical stimuli on the behavioural characteristics of nematodes.

\section{Acknowledgement}

This work was supported by the Environmental Technology Development Fund (fund number 10) from the Ministry of the Environment of Japan.

\section{References}

Brenner, K.H., Kufner, M., Kufner, S., Moisel, J., Müller, A., Sinzinger, S., Testorf, M., Gottert,
J. \& Mohr, J. (1993). Applications of three-dimensional micro-optical components formed by lithography, electroforming, and plastic molding. Applied Optics 32, 6464-6469.

CROLL, N.A. (1970). The behaviour of nematodes. London, UK, Edward Arnold Ltd, 117 pp.

Glasbey, C.A., Horgan, G.W. \& Darbyshire, J.F. (1991). Image analysis and three-dimensional modelling of pores in soil aggregates. Journal of Soil Science 42, 479-486.

Görres, J.H., Savin, M.C., Neher, D.A., Weicht, T.R. \& AMADOR, J.A. (1999). Grazing in a porous environment: 1. The effect of soil pore structure on $\mathrm{C}$ and $\mathrm{N}$ mineralization. Plant and Soil 212, 75-83.

Hassink, J., Bouwman, L.A., Zwart, K.B. \& BrusSAARD, L. (1993). Relationships between habitable pore space, soil biota and mineralization rates in grassland soils. Soil Biology \& Biochemistry 25, 47-55.

Killham, K., Amato, M. \& Ladd, J.N. (1993). Effect of substrate location in soil and soil pore-water regime on carbon turnover. Soil Biology \& Biochemistry 25, 57-62.

Pline, M. \& Dusenbery, D.B. (1987). Responses of plantparasitic nematode Meloidogyne incognita to carbon dioxide determined by video camera - computer tracking. Journal of Chemical Ecology 13, 873-888.

Robinson, A.F. (1994). Movement of five nematode species through sand subjected to natural temperature gradient fluctuations. Journal of Nematology 26, 46-58.

Robinson, A.F. (1995). Optimal release rates for attracting Meloidogyne incognita, Rotylenchulus reniformis, and other nematodes to carbon dioxide in sand. Journal of Nematology $27,42-50$.

SAnO, Z. \& NAKASONO, K. (1997). Differences in survival time of Meloidogyne incognita juveniles inside and outside of soil aggregates in an Andosol. Soil Microorganisms 49, 17.

Sharon, E., Spiegel, Y., Salomon, R. \& Curtis, R.H.C. (2002). Characterization of Meloidogyne javanica surface coat with antibodies and their effect on nematode behaviour. Parasitology 125, 177-185.

Wallace, H.R. (1958a). Movement of eelworms - 1. The influence of pore size and moisture content of the soil on the migration of larvae of the beet eelworm, Heterodera schachtii Schmidt. Annals of Applied Biology 46, 74-85.

Wallace, H.R. (1958b). Movement of eelworms - 2. A comparative study of the movement in soil of Heterodera schachtii Schmidt and of Ditylenchus dipsaci (Kuhn) Filipjev. Annals of Applied Biology 46, 86-94. 